\title{
Differential equations and moving frames
}

\author{
Odinete Renée Abib \\ Laboratoire de Mathématiques Raphaël Salem \\ UMR 6085 CNRS \\ Université de Rouen \\ Avenue de l'Université, BP.12 \\ 76801 Saint Etienne du Rouvray, FRANCE \\ renee.abib@univ-rouen.fr
}

June 19, 2021

\begin{abstract}
We shall study the foundations of the differential geometric consideration for differential equations. We show a local structure theorem. The main idea lies in the structure equations. The Lie algebra aspects of local differential equations is studied too.
\end{abstract}

\section{Introduction}

The purpose of the present paper is to study the relationship between differential equations, Pfaffian systems and geometric structures, via the method of moving frames of E. Cartan [5, 9]

Following Cartan, we deal with every differential equation as a Pfaffian system on a suitable manifold (Section 3). This is the fundamental idea of Cartan. Further, we shall consider the structure equations which are satisfied by Pfaffian systems determined by differential equations. The intergration of a given differential equation is deeply related to the structure equation associated with the differential equation. We shall show it by means of some examples.

In Section 4, we shall establish a local structure theorem (Theorem 4.1). By virtue of this theorem, differential equations can be regarded as a differential geometric structure on a manifold. In Section [6, we shall consider the Lie algebraic aspect of local differential equations; each differential Lie algebra (definition 6.1) determines locally a local differential equation (Theorem 6.1); if $g$ is a semi-simple graded Lie algebra, then $g$ has a structure of fundamental Lie algebra (Theorem 6.2). Moreover $\operatorname{sl}(2, \mathbb{R})$ has a structure of differential Lie algebra which is not fundamental.

In Section [7 we study one system, which is one of the typical examples in Cartan's paper [6], related with $G$-structures and the local automorphism group of the given system. 
The Section 2 conscerns remarks on Pfaffian systems, Cauchy characteristics and solvable systems.

In this paper, by the language differentiable we mean differentiable of class $C^{\infty}$.

I thank Marco Antonio Teixeira, Luiz San Martin, Paulo Régis Ruffino for encouragement and IMECC-UNICAMP, BRASIL for their hospitality during the preparation of this work.

\section{Cauchy characteristic system}

We begin with the preliminary remarks on Pfaffian systems. Let $M$ be a differentiable manifold. $F(M)$ denotes the ring of real-valued differentiable functions on $M$ and $\Lambda^{1}(M)$ the $F(M)$-module of all 1-forms (Pfaffian forms) on $M$. A $F(M)$-submodule $\Sigma$ of $\Lambda^{1}(M)$ is said a Pfaffian system of rank $n$ on $M$ if $\Sigma$ is generated by $n$ linearly independent Pfaffian forms $\theta^{1}, \ldots, \theta^{n}$. A submanifold $N$ of $M$ is said an integral manifold of $\Sigma$ if $i^{*} \theta=0$ for all $\theta \in \Sigma$, where $i$ denotes the inclusion $N \hookrightarrow M$. A differentiable function $f$ on $M$ is said a first integral of $\Sigma$ if the exterior derivative $d f$ belongs to $\Sigma$. By the symbol $\Sigma=\left\langle\theta^{1}, \ldots, \theta^{n}\right\rangle$ we mean that the Pfaffian system $\Sigma$ is generated by the linearly independent Pfaffian forms $\theta^{1}, \ldots, \theta^{n}$ defined on $M$.

For each Pfaffian system $\Sigma$ on $M$, we can construct the dual system, that is, the differentiable subbundle $D(\Sigma)$ of the tangent bundle $T(M)$ on $M$ such that the fiber dimension of $D(\Sigma)$ is equal to $\operatorname{dim} M-n$. Let $D(\Sigma)$ be the sheaf of germs of local vector fields which belong to $D(\Sigma)$ and $\underline{D(\Sigma)} x$ (x $(x \in \overline{M)}$ the stalk of $\underline{D(\Sigma)}$ at $x$. We set

$$
{\underline{\operatorname{Ch}(D(\Sigma))_{x}}}_{x}=\left\{A \in \underline{D(\Sigma)}_{x} \mid\left[A, \underline{D(\Sigma)}{ }_{x}\right] \subset \underline{D(\Sigma)}_{x}\right\}
$$

where [, ] denotes the natural bracket operation. Further, for each $x \in M$, we define the subspaces $\mathrm{Ch}(D(\Sigma))_{x}$ of $T_{x}(M)$ by

$$
\operatorname{Ch}(D(\Sigma))_{x}=\left\{X_{x} \in D(\Sigma)_{x} \mid \underline{X}_{x} \in \underline{\operatorname{Ch}(D(\Sigma))_{x}}\right\},
$$

where $X$ denotes a vector field and $\underline{X}_{x}$ the germ at $x$ determined by $X$. We suppose that $\operatorname{dim} \operatorname{Ch}(D(\Sigma))_{x}$ is constant on $M$. Thus, we obtain the subbundle $\operatorname{Ch}(D(\Sigma))$ of $T(M)$. $\operatorname{Ch}(D(\Sigma))$ is called the Cauchy charateristic of $D(\Sigma)$. The dual system of $\operatorname{Ch}(D(\Sigma))$ is called the Cauchy characteristic system of $\Sigma$. The following theorem is due to Cartan [3, 4.

Theorem 2.1 Let $\Sigma=\left\langle\theta^{1}, \ldots, \theta^{n}\right\rangle$ be a Pfaffian system.

1. If $\Sigma$ is completely integrable, i.e. $d \theta^{i}=0\left(\bmod . \theta^{1}, \ldots, \theta^{n}\right) i=1,2, \ldots, n$, then $\operatorname{Ch}(\Sigma)=\Sigma$.

2. If $\Sigma$ is not completely integrable, then there exist linearly independent Pfaffian forms $\omega^{1}, \ldots, \omega^{m}$ satisfying the following conditions:

(i) $\theta^{1}, \ldots, \theta^{n}, \omega^{1}, \ldots, \omega^{m}$ are also linearly independent; 
(ii) $\left(\theta^{1}, \ldots, \theta^{n}, \omega^{1}, \ldots, \omega^{m}\right)$ forms a (local) generator of $\mathrm{Ch}(\Sigma)$;

(iii) $d \theta^{i}=\sum_{j, k=1}^{m} C_{j k}^{i} \omega^{j} \wedge \omega^{k}$ (mod. $\left.\theta^{1}, \ldots, \theta^{n}\right)$, where $C_{j k}^{i}$ denotes a differentiable function $(i=1,2, \ldots, n ; j, k=1,2, \ldots, m)$.

3. $\mathrm{Ch}(\Sigma)$ is completely integrable.

4. Let $x^{1}, \ldots, x^{n+m}$ be independent first integrals of $\mathrm{Ch}(\Sigma)$. Then there exist linearly independent Pfaffian forms $\bar{\theta}^{i}=\sum_{j=1}^{n+m} A_{j}^{i}\left(x^{1}, \ldots, x^{n+m}\right) d x^{j}, i=1,2, \ldots, n$, such that $\left(\bar{\theta}^{1}, \ldots, \bar{\theta}^{n}\right)$ forms a (local) generator of $\Sigma$.

By making use of property 2.(ii), we can construct the Cauchy characteristic system $\operatorname{Ch}(\Sigma)$.

Example 2.1 Consider the Pfaffian system $\Sigma=\langle\theta\rangle, \theta=d z+p d x+p^{2} d y$, on $\mathbb{R}^{4}=$ $\{(x, y, z, p)\}$. We have dtheta $=d p \wedge(d x+2 p d y)$ and

$$
\omega^{1}=d p \quad, \quad \omega^{2}=d x+2 p d y \quad, \quad \omega^{3}=p \quad ;
$$

determine the Cauchy characteristic system of $\Sigma$. We can find by quadrature three independent first integrals as follows:

$$
u_{1}=z+x p+y p^{2} \quad, \quad u_{2}=x+2 y p \quad, \quad u_{3}=p \quad ;
$$

and $\theta$ itself is expressed as $\theta=d u_{1}-u_{2} d u_{3}$.

Definition 2.1 A system $\left(\omega^{1}, \ldots, \omega^{m}\right)$ of linearly independent Pfaffian forms on $M$ will be said a solvable system of $\Sigma=\left\langle\theta^{1}, \ldots, \theta^{n}\right\rangle$ if it satisfies the following conditions:

(i) $\left(\omega^{1}, \ldots, \omega^{m}\right)$ forms a generator of $\mathrm{Ch}(\Sigma)$;

(ii) $d \omega^{1}=0$ and $d \omega^{p} \equiv 0$ (mod. $\left.\omega^{1}, \ldots, \omega^{p-1}\right)$ for all $p=2,3, \ldots, m$.

If we can find a solvable system of $\Sigma$, then $m$ independent first integrals of $\mathrm{Ch}(\Sigma)$ are given by quadrature. In the above example, the system $\left(\omega^{1}, \omega^{2}, \omega^{3}\right)$ is a solvable system of $\Sigma=\langle\theta\rangle$.

\section{Differential equations and structure equations}

In this section we shall consider, by means of simple examples, the relation between the differential equations and Pfaffian systems. 
a) Take the first order equation on $\mathbb{R}^{2}=\{(x, y)\}$

$$
\frac{\partial z}{\partial x}+\frac{1}{2}\left(\frac{\partial z}{\partial y}\right)^{2}=0
$$

Setting on $\mathbb{R}^{4}=\{(x, y, z, q)\}, \omega^{1}=d x, \omega^{2}=d y-q d x, \omega^{3}=d z+\frac{1}{2} q^{2} d y-q d y, \omega^{4}=d q$, we have

$$
\left\{\begin{array}{l}
d \omega^{1}=0 \\
d \omega^{2}=\omega^{1} \wedge \omega^{4} \\
d \omega^{3}=\omega^{2} \wedge \omega^{4} \\
d \omega^{4}=0
\end{array}\right.
$$

Each integral of (3.1) defines a 2-dimensional integral manifold of $\left\langle\omega^{3}\right\rangle$ on which $\omega^{1}$ and $\omega^{2}$ are linearly independent. The equation (3.1) is left invariant by the automorphism group of the absolute parallelism $\omega^{1}, \ldots, \omega^{4}$ on $\mathbb{R}^{4}$. The structure of this group is determined by the equation (3.2). The integration of the equation (3.1) depends deeply on the structure equation (3.2) of this group. In this case $\left(\omega^{1}, \omega^{2}, \omega^{3}\right)$ forms a solvable system of $\left\langle\omega^{3}\right\rangle$. Therefore three independent first integrals of $\mathrm{Ch}\left(\left\langle\omega^{3}\right\rangle\right)$ are given by quadrature as

$$
u_{1}=q \quad, \quad u_{2}=z+\frac{1}{2} x q^{2}-y q \quad, \quad u_{3}=y-x q \quad ;
$$

and we have $\omega^{3}=d u_{2}+u_{3} d u_{1}$. The formula

$$
\left\{\begin{array}{l}
z+\frac{1}{2} x q^{2}-y q=f(q) \\
y-x q+f^{\prime}(q)=0
\end{array}\right.
$$

gives an integral surface of the equation (3.1), where $f$ is a differentiable function and $f^{\prime}$ denotes its derivative.

Conversely, we consider an absolute parallelism $\omega^{1}, \omega^{2}, \omega^{3}, \omega^{4}$ on $\mathbb{R}^{4}$ satisfying the equations

$$
\left\{\begin{array}{l}
d \omega^{1} \equiv 0, d \omega^{2} \equiv 0 \quad\left(\bmod . \omega^{1}, \omega^{2}\right) \\
d \omega^{3} \equiv \omega^{2} \wedge \omega^{4} \quad\left(\bmod . \omega^{3}\right)
\end{array}\right.
$$

Let $x$ and $y$ be two independent first integals of the completely integrable Pfaffian system $\omega^{1}=\omega^{2}=0$. If we reduce $\omega^{3}$ to the submanifold defined by the equations $x=$ const., $y=$ const., then from the equation $d \omega^{3} \equiv \omega^{2} \wedge \omega^{4}\left(\bmod . \omega^{3}\right)$ we have $d \omega^{3} \equiv 0\left(\bmod . \omega^{3}\right)$ on this submanifold. Therefore $\omega^{3}$ must be of the form

$$
\omega^{3}=a(d z-p d x-q d y)
$$

where $a$ is a non-zero function. Since $\omega^{1}, \omega^{2}, \omega^{3}$ are linearly independent, the functions $x, y$ and $z$ are also independent.

By this procedure we can determine the functions $p$ and $q$ of the variables $x, y, z$ and another $t$ :

$$
p=p(x, y, z, t) \quad, \quad q=q(x, y, z, t)
$$

and the same equation $d \omega^{3} \equiv \omega^{2} \wedge \omega^{4}\left(\bmod . \omega^{3}\right)$ implies

$$
\operatorname{rank}\left(\frac{\partial p}{\partial t}, \frac{\partial q}{\partial t}\right)=1
$$


On a 2-dimensional integral manifold of $\omega^{3}=0$ on which $x$ and $y$ are still independent, $p$ and $q$ can be considered as the first partial derivatives of $z=z(x, y)$. Therefore the equation (3.4) can be regarded as a first-order differential equation. For example, the differential equation

$$
\frac{\partial z}{\partial x}+\frac{1}{2}\left(\frac{\partial z}{\partial y}-f(x, y)\right)^{2}=g(x, y)
$$

belongs to the family determined by the structure equation (3.2), where $f(x, y)$ and $g(x, y)$ are differentiable functions satisfying the equation

$$
\frac{\partial f}{\partial x}=\frac{\partial g}{\partial y} .
$$

b) Next, we consider an absolute parallelism $\omega^{1}, \omega^{2}, \omega^{3}, \omega^{4}, \omega^{5}, \omega^{6}$ on $\mathbb{R}^{6}$ satisfying

$$
\left\{\begin{array}{l}
d \omega^{1} \equiv 0, d \omega^{2} \equiv 0 \quad\left(\bmod . \omega^{1}, \omega^{2}\right) \\
d \omega^{3} \equiv \omega^{1} \wedge \omega^{4}+\omega^{2} \wedge \omega^{5} \quad\left(\bmod . \omega^{3}\right) \\
d \omega^{4}=0 \quad\left(\bmod . \omega^{3}, \omega^{4}, \omega^{5}\right) \\
d \omega^{5}=\omega^{2} \wedge \omega^{6} \quad\left(\bmod . \omega^{3}, \omega^{4}, \omega^{5}\right) .
\end{array}\right.
$$

Let $x$ and $y$ be two independent first integrals of the completely Pfaffian integrable system $\omega^{1}=\omega^{2}=0 ; \omega^{3}$ is expressed as

$$
\omega^{3}=a(d z-p d x-q d y) \quad(a \neq 0) .
$$

The functions $x, y, z, p$ and $q$ are independent first integrals of the completely integrable Pfaffian system $\omega^{1}=\omega^{2}=\omega^{3}=\omega^{4}=\omega^{5}=0$. Therefore $\omega^{4}$ and $\omega^{5}$ can be written by means of the exterior derivatives $d x, d y, d z, d p, d q$ and the formulas

$$
\begin{aligned}
& d p-r d x-s d y=a_{1} \omega^{4}+a_{2} \omega^{5}+a_{3} \omega^{3} \\
& d q-s^{\prime} d x-t d y=a_{4} \omega^{4}+a_{5} \omega^{5}+a_{6} \omega^{3}
\end{aligned}
$$

determine the functions $r, s, s^{\prime}, t$ and $a_{i}$ 's of the variables $x, y, z, p, q$ and another $u$. From the equation $d \omega^{3} \equiv \omega^{1} \wedge \omega^{4}+\omega^{2} \wedge \omega^{5}\left(\bmod . \omega^{3}\right)$, one can verify that the function $s$ coincides with $s^{\prime}$. Moreover, the equations $d \omega^{4}=0 \quad, d \omega^{5}=\omega^{2} \wedge \omega^{6}\left(\bmod . \omega^{3}, \omega^{4}, \omega^{5}\right)$ imply

Therefore the functions

$$
\operatorname{rank}\left(\frac{\partial r}{\partial u}, \frac{\partial s}{\partial u}, \frac{\partial t}{\partial u}\right)=1
$$

$$
r=r(x, y, z, p, q, u), s=s(x, y, z, p, q, u), t=t(x, y, z, p, q, u)
$$

determine a system of second-order partial differential equations. This family of systems of differential equations determined by an absolute parallelism satisfying (3.5) is the main subject of Cartn's researches in his paper [6].

For example, take the system of differential equations (c.f. [6, $\S \S 13,14]$ )

$$
\frac{\partial^{2} z}{\partial x^{2}}=0 \quad, \quad \frac{\partial^{2} z}{\partial x \partial y}=z-x \frac{\partial z}{\partial x}
$$


Putting on $\mathbb{R}^{6}=\{(x, y, z, p, q, t)\} \omega^{1}=d x, \omega^{2}=d y, \omega^{3}=d z-p d x-q d y, \omega^{4}=$ $d p-(z-x p) d y, \omega^{5}=d q-(z-x p) d x-t d y$ and $\omega^{6}=d t-(q-x(z-x p)) d x$ we have the structure equations

$$
\left\{\begin{array}{l}
d \omega^{1} \equiv 0, d \omega^{2} \equiv 0 \\
d \omega^{3} \equiv \omega^{1} \wedge \omega^{4}+\omega^{2} \wedge \omega^{5} \\
d \omega^{4} \equiv \omega^{2} \wedge \omega^{3}-x \omega^{2} \wedge \omega^{4} \\
d \omega^{5}=\omega^{2} \wedge \omega^{6}+\omega^{1} \wedge \omega^{3}-x \omega^{1} \wedge \omega^{4} \\
d \omega^{6}=\omega^{1} \wedge \omega^{5}-x \omega^{1} \wedge \omega^{3}-x^{2} \omega^{1} \wedge \omega^{4}+K \omega^{1} \wedge \omega^{2}
\end{array}\right.
$$

where $K=t-x q+x^{2}(z-x p)$. The absolute parallelism satisfies the equations (3.5). It is easy to see that the system $\left(\omega^{2}, \omega^{3}, \omega^{4}, \omega^{5}, \omega^{6}\right)$ forms a solvable system of $\Sigma=\left\langle\omega^{3}, \omega^{4}, \omega^{5}\right\rangle$. Five independent first integrals of the solvable system are given by quadrature as follows:

$$
u_{1}=y \quad, \quad u_{2}=z-x p \quad, \quad u_{3}=p \quad, \quad u_{4}=q-x(z-x p) \quad, \quad u_{5}=K \quad
$$

and we have (c.f. [6, $\S 10$, IV])

$$
\left\{\begin{aligned}
\omega^{3}-x \omega^{4} & =d u_{2}-u_{4} d u_{1} \\
\omega^{4} & =d u_{3}-u_{2} d u_{1} \\
\omega^{5}-x \omega^{3} & =d u_{4}-u_{5} d u_{1}
\end{aligned}\right.
$$

By this expression, the general integral surface of (3.6) is given by the formulas:

$$
p=f(y), z-x p=f^{\prime}(y), q-x(z-x p)=f^{\prime \prime}(y), t-x(q-x(z-x p))=f^{\prime \prime \prime}(y)
$$

where $f$ is a differentiable function and $f^{\prime}, f^{\prime \prime}$ and $f^{\prime \prime \prime}$ denote its derivatives.

\section{Differential geometric structures}

In the previous section we have seen that the integration of differential equation is deeply related to the structure equations of differential equations. In this section we shall consider the differential geometric structures for differential equations.

Let $V_{-1}$ and $V_{0}$ be finite dimensional real vector spaces. We define by induction the real vector spaces $V_{k}, k=1,2, \ldots$ as follows. Let $V_{1}=\operatorname{Hom}\left(V_{-1}, V_{0}\right) ; V_{k-1}(k \geq 2)$ being determined, we set

$$
V_{k}=\left\{X \in \operatorname{Hom}\left(V_{-1}, V_{k-1}\right) \mid X(u)(v)=X(v)(u), u, v, \in V_{-1}\right\} .
$$

We have $V_{k} \cong V_{0} \otimes S^{k}\left(V_{-1}^{*}\right)$ as a vector space $(k=0,1,2, \ldots)$, where $S^{k}\left(V_{-1}^{*}\right)$ denotes the symmetric tensor space of the dual space $V_{-1}^{*}$. For an integer $k \geq 1$ we set

$$
W_{k}\left(V_{-1}, V_{0}\right)=V_{-1} \oplus V_{0} \oplus \cdots \oplus V_{k} \quad \text { (direct sum), }
$$

and we define the bracket operation $[$,$] on W_{k}\left(V_{-1}, V_{0}\right)$ as follows: 
(i) For all $X_{-1} \in V_{-1}, X_{p} \in V_{p}(p \geq 1)$,

$$
\left[X_{p}, X_{-1}\right]=-\left[X_{-1}, X_{p}\right]=X_{p}\left(X_{-1}\right)
$$

(ii) $[X, Y]=0$ for any other combination

By this bracket operation, $W_{k}\left(V_{-1}, V_{0}\right)$ becomes a nilpotent Lie algebra. It is easy to prove the following.

\section{Proposition 4.1}

(i) For a non-zero element $X_{-1} \in V_{-1},\left[X_{-1}, V_{p}\right]=V_{p-1}(p \geq 1)$.

(ii) If $\left[X_{-1}, V_{p}\right]=(0)$ for $X_{p} \in V_{p}(p \geq 1)$, then $X_{p}=0$.

(iii) For an arbitrary subspace $V_{k}^{0}$ of $V_{k}$,

$$
W_{k}^{0}\left(V_{-1}, V_{0}\right)=V_{-1} \oplus V_{0} \oplus \cdots \oplus V_{k}^{0} \quad \text { (direct sum) }
$$

is a Lie subalgebra of $W_{k}\left(V_{-1}, V_{0}\right)$.

\section{Example 4.1}

(1) $\operatorname{dim} V_{-1}=1, \operatorname{dim} V_{0}=1$. We have $\operatorname{dim} V_{k}=1$ for any $k \geq 1$. There exists a basis $X_{-1}, X_{0}, X_{1}, \ldots, X_{k}$ of $W_{k}\left(V_{-1}, V_{0}\right)$ such that $X_{p} \in V_{p}(-1 \leq p \leq k)$ and $\left[X_{-1}, X_{p}\right]=-X_{p-1}(1 \leq p \leq k)$.

(2) $\operatorname{dim} V_{-1}=2, \operatorname{dim} V_{0}=1$. We have $\operatorname{dim} V_{k}=k+1$ for any $k \geq 1$.

(i) $k=1$. There exists a basis $X_{1}, X_{2}, X_{3}, X_{4}, X_{5}$ of $W_{k}\left(V_{-1}, V_{0}\right)$ such that $X_{1}, X_{2} \in V_{-1} ; X_{3} \in V_{0} ; X_{4}, X_{5} \in V_{1}$ and

$$
\left[X_{1}, X_{4}\right]=-X_{3} \quad, \quad\left[X_{2}, X_{5}\right]=-X_{3}
$$

and otherwise $[X, Y]=0$.

(ii) $k=2$. There exists a basis $X_{1}, X_{2}, X_{3}, X_{4}, X_{5}, X_{6}, X_{7}, X_{8}$ of $W_{2}\left(V_{-1}, V_{0}\right)$ such that $X_{1}, X_{2} \in V_{-1} ; X_{3} \in V_{0} ; X_{4}, X_{5} \in V_{1} ; X_{6}, X_{7}, X_{8} \in V_{2}$ and

$$
\begin{array}{lllll}
{\left[X_{1}, X_{4}\right]=-X_{3}} & , & {\left[X_{2}, X_{5}\right]=-X_{3}} & , & {\left[X_{1}, X_{6}\right]=-X_{4}} \\
{\left[X_{1}, X_{7}\right]=-X_{5}} & , & {\left[X_{2}, X_{7}\right]=-X_{4}} & , & {\left[X_{2}, X_{8}\right]=-X_{5}}
\end{array}
$$

and otherwise $[X, Y]=0$.

Let $\pi: M \rightarrow N$ be a fibered manifold on a differentiable manifold $N$ and $J^{k}(M, \pi)$ the space of $k$-jets of local sections of $\pi$. If $\operatorname{dim} N=\operatorname{dim} V_{-1}$ and $\operatorname{dim} M=\operatorname{dim}\left(V_{-1} \oplus V_{0}\right)$, $W_{k}\left(V_{-1}, V_{0}\right)$ is regarded as the local structure of $J^{k}(M, \pi)$, i.e. $J^{k}(M, \pi) \cong W_{k}\left(V_{-1}, V_{0}\right)$ (locally diffeomorphic). 
Let $u^{p}: W_{k}\left(V_{-1}, V_{0}\right) \rightarrow V_{p}(-1 \leq p \leq k)$ be the natural projection. We regard $u^{p}$ as a vector-space valued function on $W_{k}\left(V_{-1}, V_{0}\right)$, so that the system $\left(u^{-1}, u^{0}, \ldots, u^{k}\right)$ can be considered as a linear coordinate system on $W_{k}\left(V_{-1}, V_{0}\right)$. We set $\theta^{-1}=d u^{-1}$, $\theta^{p}=d u^{p}-\left[u^{p-1}, d u^{-1}\right](0 \leq p \leq k-1), \theta^{k}=d u^{k}$ and $\theta=\theta^{-1}+\theta^{0}+\cdots+\theta^{k}$. $\theta$ is a $W_{k}\left(V_{-1}, V_{0}\right)$-valued 1-form on $W_{k}\left(V_{-1}, V_{0}\right)$. We have

$$
\left\{\begin{array}{l}
d \theta^{-1}=0 \\
d \theta^{p}+\left[\theta^{-1} \wedge \theta^{p+1}\right]=0 \quad(0 \leq p \leq k-1) .
\end{array}\right.
$$

For example, making use of the notations in Example 4.1 2.(ii), we set $u^{-1}=x X_{1}+y X_{2}$, $u^{0}=z X_{3}, u^{1}=p X_{4}+q X_{5}, u_{2}=r X_{6}+s X_{7}+t X_{8}$ and $\theta=\sum_{i=1}^{8} \omega^{i} X_{i}$. Then we have $\omega^{1}=d x, \omega^{2}=d y, \omega^{3}=d z-p d x-q d y, \omega^{4}=d p-r d x-s d y, \omega^{5}=d q-s d x-t d y$ $\omega^{6}=d r, \omega^{7}=d s, \omega^{8}=d t$ and

$$
\left\{\begin{array}{l}
d \omega^{1}=0, d \omega^{2}=0 \\
d \omega^{3}=\omega^{1} \wedge \omega^{4}+\omega^{2} \wedge \omega^{5} \\
d \omega^{4}=\omega^{1} \wedge \omega^{6}+\omega^{2} \wedge \omega^{7} \\
d \omega^{5}=\omega^{1} \wedge \omega^{7}+\omega^{2} \wedge \omega^{8} .
\end{array}\right.
$$

Let $\rho^{k}: W_{k}\left(V_{-1}, V_{0}\right) \rightarrow W_{k-1}\left(V_{-1}, V_{0}\right)(k \geq 1)$ be the natural projection, where we put $W_{0}\left(V_{-1}, V_{0}\right)=V_{-1} \oplus V_{0} ; W_{k}\left(V_{-1}, V_{0}\right)$ can be considered as a fibered manifold on $W_{k-1}\left(V_{-1}, V_{0}\right)$ with the fibering $\rho^{k}$.

Definition 4.1 We shall say that a submanifold $R_{k}$ of $W_{k}\left(V_{-1}, V_{0}\right)$ is a local differential equation of order $k$ if $R_{k}$ admits an absolute parallelism and if there exist an open submanifold $U$ of $W_{k-1}\left(V_{-1}, V_{0}\right)$ such that $\rho^{k}\left(R_{k}\right)=U$ and $\left.\rho^{k}\right|_{R_{k}}: R_{k} \rightarrow U$ is a fibered submanifold of $\rho^{k}:\left(\rho^{k}\right)^{-1}(U) \rightarrow U$.

Let $i: R_{k} \hookrightarrow W_{k}\left(V_{-1}, V_{0}\right)$ be the inclusion and $\omega=i^{*} \theta$ the induced $W_{k}\left(V_{-1}, V_{0}\right)$-valued 1-form on $R_{k}$. According to the direct sum decomposition of $W_{k}\left(V_{-1}, V_{0}\right)$, we decompose $\omega$ as $\omega=\omega^{-1}+\omega^{0}+\cdots \widetilde{\omega}^{k}$, where $\omega^{p}$ (resp. $\left.\widetilde{\omega}^{k}\right)$ is a $V_{p^{-}}$valued 1-form (resp. $V_{k}$-valued 1-form) on $R_{k}(-1 \leq p \leq k-1)$. Let $n$ be the fiber dimension of $\rho: R_{k} \rightarrow U$. Then there exist $n$ linearly independent Pfaffian forms $\omega_{1}^{k}, \ldots, \omega_{n}^{k}$ which are also linearly independent of the Pfaffian forms obtained from $\omega^{-1}, \omega^{0}, \ldots, \omega^{k-1}$. We fix a $n$-dimensional vector subspace $V_{k}^{0}$ of $V_{k}$ and its basis $X_{1}, \ldots, X_{n}$ and we set $\omega^{k}=\sum_{j=1}^{n} \omega_{j}^{k} X_{j}$. $\omega^{k}$ is a $V_{k}^{0}$-valued 1-form on $R_{k}$. We define the differentiable mappings

$$
\begin{array}{ll}
F_{p}^{k}: & R_{k} \longrightarrow \operatorname{Hom}\left(V_{p}, V_{k}\right) \\
F_{k}^{k} & : \quad R_{k} \longrightarrow \operatorname{Hom}\left(V_{k}^{0}, V_{k}\right)
\end{array} \quad(-1 \leq p \leq k-1),
$$

by the formula

$$
d\left(u^{k} \circ i\right)=F_{-1}^{k}\left(\omega^{-1}\right)+\cdots+F_{k}^{k}\left(\omega^{k}\right) ;
$$

and we define the differentiable mapping

$$
T: R_{k} \longrightarrow \operatorname{Hom}\left(V_{-1} \times V_{k}^{0}, V_{k-1}\right)
$$


by the formula

$$
T_{x}\left(X_{-1}, X_{k}\right)=\left[X_{-1}, F_{k}^{k}(x)\left(X_{k}\right)\right] \quad\left(x \in R_{k}, X_{-1} \in V_{-1}, X_{k} \in V_{k}^{0}\right) .
$$

Since the rank of the inclusion $i$ is maximal on $R_{k}$, the linear mapping $F_{k}^{k}(x): V_{k}^{0} \rightarrow V_{k}$ $\left(x \in R_{k}\right)$ is injective. Therefore $T$ has the following property:

$\left(\mathrm{C}_{1}\right)$ For each $x \in R_{k}, T_{x}\left(V_{-1}, X_{k}\right)=0\left(X_{k} \in V_{k}^{0}\right)$ implies $X_{k}=0$.

It is also easy to prove the following properties:

(i) $d \omega^{-1} \equiv 0\left(\bmod . \omega^{-1}\right)$;

(ii) $(k \geq 2)$ For $p=0,1, \ldots, k-2$,

$$
d \omega^{p}+\left[\omega^{-1} \wedge \omega^{p-1}\right] \equiv 0 \quad\left(\bmod . \omega^{0}, \ldots, \omega^{p}\right)
$$

(iii) $d \omega^{k-1}+T\left(\omega^{-1} \wedge \omega^{k}\right) \equiv 0\left(\bmod . \omega^{-1} \wedge \omega^{-1}, \omega^{0}, \ldots, \omega^{k-1}\right)$.

We have thus proved that for each local differential equation of order $k$ there exist a differentiable mapping $T: R_{k} \rightarrow \operatorname{Hom}\left(V_{-1} \times V_{k}^{0}: V_{k}\right)$ and an absolute parallelism $\omega=\omega^{-1}+\omega^{0}+\cdots \omega^{k}$ satisfying the above conditions $\left(\mathrm{C}_{1}\right),\left(\mathrm{C}_{2}\right)$.

Theorem 4.1 Let $V_{k}^{0}$ be a subspace of $V_{k}$ and put $W_{k}^{0}=V_{-1} \oplus V_{0} \oplus \cdots \oplus V_{k}^{0}$ (direct sum). Let $R_{k}$ be a differentiable manifold with $\operatorname{dim} R_{k}=\operatorname{dim} W_{k}^{0}$. If there exists a $W_{k}^{0}$-valued absolute parallelism $\omega=\omega^{-1}+\omega^{0}+\cdots \omega^{k}$ and a differentiable mapping $T$ : $R_{k} \rightarrow \operatorname{Hom}\left(V_{-1} \times V_{k}^{0}: V_{k}\right)$ satisfying the conditions $\left(C_{1}\right)$ and $\left(C_{2}\right)$, then $R_{k}$ can be locally embedded into $W_{k}\left(V_{-1}, V_{0}\right)$ as a local differential equation or order $k$.

ProOF. Since $\omega$ gives rise to an isomorphism $\omega_{x}: T_{x}\left(R_{k}\right) \stackrel{\sim}{\rightarrow} W_{k}^{0}\left(x \in R_{k}\right)$ one can consider the inverse mapping of $\omega_{x}$, say $\tau_{x}: W_{k}^{0} \stackrel{\sim}{\rightarrow} T_{x}\left(R_{k}\right) . \tau$ has the property: $\omega^{p}\left(\left(\tau\left(X_{q}\right)\right)=\delta_{q}^{p} X_{q}\right.$, $X_{q} \in V_{q}(-1 \leq p, q \leq k)$. By the condition (i) of $\mathrm{C}_{2}$, we can find differentiable mappings $v^{-1}: R_{k} \rightarrow V_{-1}$ and $A_{-1}: R_{k} \rightarrow \mathrm{GL}\left(V_{-1}\right)$ such that $\omega^{-1}=A_{-1}\left(d v^{-1}\right)$. Since the system $\omega^{-1}=\omega^{0}=0$ is completely integrable, there exists a differentiable mapping $v^{0}: R_{k} \rightarrow V_{0}$ such that the system $d v^{-1}=d v^{0}=0$ is equivalent to the system $\omega^{-1}=\omega^{0}=0$. Therefore, $\omega^{0}$ can be written as

$$
\omega^{0}=A_{0}\left(d v^{0}-v^{1} d v^{-1}\right),
$$

where $A_{0}$ denotes a differentiable mapping $A_{0}: R_{k} \rightarrow \mathrm{GL}\left(V_{0}\right)$ and $v^{1}$ denotes a differentiable mapping $v^{1}: R_{k} \rightarrow \operatorname{Hom}\left(V_{-1}, V_{0}\right)=V_{1}$. If $k=1$ the argument comes to an end. Let $k \geq 2$. Consider the following proposition $\left(\mathrm{P}_{j}\right)$ for $1 \leq j \leq k$ :

$\left(\mathrm{P}_{j}\right)$ There exist differentiable mappings $v^{-p}: R_{k} \rightarrow V_{p}$ and $A_{p}: R_{k} \rightarrow \mathrm{GL}\left(V_{p}\right), p=$ $-1,0,1, \ldots j$, such that

(1) $d v^{-1}, d v^{0}, \ldots, d v^{j-1}$ are linearly independent;

(2) $\omega^{-1}=A_{-1}\left(d v^{-1}\right), \omega^{0}=A_{0}\left(d v^{0}-v^{1} d v^{-1}\right)$ and for $p=1,2, \ldots, j-1$, $\omega^{p}=A_{p}\left(d v^{p}-v^{p-1} d v^{-1}\right)\left(\bmod \cdot \omega^{0}, \ldots, \omega^{p-1}\right)$. 
We have proved $\left(\mathrm{P}_{1}\right)$. For an integer $1 \leq j \leq k-1$, assume that $\left(\mathrm{P}_{j}\right)$ is established. From the inequality $0 \leq j-1 \leq k-2$ and condition (ii) of $\left(\mathrm{C}_{2}\right)$ we have

$$
d \omega^{j-1} \equiv-\left[\omega^{-1} \wedge \omega^{j}\right] \quad\left(\bmod . \omega^{0}, \ldots, \omega^{j-1}\right)
$$

From $(2)$ of $\left(\mathrm{P}_{j}\right)$ we have

$$
d \omega^{j-1} \equiv-A_{j-1}\left(d v j \wedge d v^{-1}\right) \quad\left(\bmod . \omega^{0}, \ldots, \omega^{j-1}\right)
$$

These two equations yield

$$
-A_{j-1}\left(d v j \wedge d v^{-1}\right) \equiv-\left[\omega^{-1} \wedge \omega^{j}\right] \quad\left(\bmod . \omega^{0}, \ldots, \omega^{j-1}\right) .
$$

Substituting $\tau\left(X_{p}\right) \wedge \tau\left(X_{-1}\right), X_{-1} \in V_{-1}, X_{p} \in V_{p}(j+1 \leq p \leq k)$ to this equation, we obtain

$$
\left(d v^{j}\left(\tau\left(X_{p}\right)\right)\right)\left(A_{-1}^{-1}\left(X_{-1}\right)\right)=0
$$

and hence

$$
d v^{j}\left(\tau\left(X_{p}\right)\right)=0 \quad\left(X_{p} \in V_{p}: p=j+1, \ldots, k\right)
$$

Substituting $\tau\left(X_{j}\right) \wedge \tau\left(X_{-1}\right), X_{-1} \in V_{-1}, X_{j} \in V_{j}$ to equation (4.1), we have

$$
A_{j-1}\left(d v^{j}\left(\tau\left(X_{j}\right)\right)\right)\left(A_{-1}^{-1}\left(X_{-1}\right)\right)=-\left[X_{-1}, X_{j}\right]
$$

and hence

$$
d v^{j}\left(\tau\left(X_{j}\right)\right)=A_{j-1}^{-1} \circ X_{j} \circ A_{-1}
$$

The equation (4.2) implies that $d v^{j}$ is expressed as

$$
d v^{j}=B_{j}\left(\omega^{j}\right) \quad\left(\bmod \cdot \omega^{-1}, \omega^{0}, \ldots, \omega^{j-1}\right)
$$

and the equation (4.3) implies that the differentiable mapping $B_{j}: R_{k} \rightarrow \operatorname{Hom}\left(V_{j}, V_{j}\right)$ is given by the formula

$$
B_{j}\left(X_{j}\right)=A_{j-1}^{-1} \circ X_{j} \circ A_{-1} \quad\left(X_{j} \in V_{j}\right)
$$

so that $B_{j}(x)$ is non-singular for any $x \in R_{k}$. Therefore one can see that $d v^{-1}, d v^{0}, \ldots, d v^{j}$ are linearly independent. By equation (4.4), $\omega^{j}$ can be written as

$$
\left.\omega^{j}=A_{j}\left(d v^{j}-v^{j+1} d v^{-1}\right) \quad \bmod . \omega^{0}, \ldots, \omega^{j-1}\right) .
$$

where $A_{j}=B_{j}^{-1}$ and $v^{j+1}$ denotes a differentiable mapping $v^{j+1}: R_{k} \rightarrow \operatorname{Hom}\left(V_{-1}, V_{j}\right)$. Substituting $\tau\left(X_{-1}\right), X_{-1} \in V_{-1}$, to this equation, we have

$$
d v^{j}\left(\tau\left(X_{-1}\right)\right)=v^{j+1}\left(A_{-1}^{-1}\left(X_{-1}\right)\right) .
$$

Substituting $\tau\left(X_{-1}\right) \wedge \tau\left(Y_{-1}\right), X_{-1}, Y_{-1} \in V_{-1}$, to equation (4.1), we obtain

$$
d v^{j}\left(\tau\left(X_{-1}\right)\right)\left(A_{-1}^{-1}\left(Y_{-1}\right)\right)=d v^{j}\left(\tau\left(Y_{-1}\right)\right)\left(A_{-1}^{-1}\left(X_{-1}\right)\right)
$$


These two equations imply

$$
v^{j+1}\left(X_{-1}\right)\left(Y_{-1}\right)=v^{j+1}\left(Y_{-1}\right)\left(X_{-1}\right)
$$

for any $X_{-1}, Y_{-1} \in V_{-1}$, so that $v^{j+1}(x)$ lies in $V_{j+1}$ for any $x \in R_{k}$. Thus we can establish by induction the proposition $\left(\mathrm{P}_{j+1}\right)$ and hence $\left(\mathrm{P}_{k}\right)$. Define the differentiable mapping $F: R_{k} \rightarrow W_{k}\left(V_{-1}, V_{0}\right)$ by the formula

$$
u^{p} \circ F=v^{p} \quad(-1 \leq p \leq k)
$$

and put

$$
d v^{k} \equiv v_{k}^{k}\left(\omega^{k}\right) \quad\left(\bmod \cdot \omega^{-1}, \omega^{0}, \ldots, \omega^{k-1}\right)
$$

If $v_{k}^{k}(x) \in \operatorname{Hom}\left(V_{k}^{0}, V_{k}\right)$ is injective for any $x \in R_{k}, F$ is an immersion and determines locally an embedding. From the proposition $\left(\mathrm{P}_{k}\right)$ we have

$$
\begin{array}{rlr}
d \omega^{k-1} & \equiv-A_{k-1}\left(d v^{k} \wedge A_{-1}^{-1}\left(\omega^{-1}\right)\right) \quad\left(\bmod . \omega^{0}, \ldots, \omega^{k-1}\right) \\
& \equiv-A_{k-1}\left(v_{k}^{k}\left(\omega^{k}\right) \wedge A_{-1}^{-1}\left(\omega^{-1}\right)\right) \quad\left(\bmod \cdot \omega^{-1} \wedge \omega^{-1}, \omega^{0}, \ldots, \omega^{k-1}\right) .
\end{array}
$$

By the condition (iii) of $\left(\mathrm{C}_{2}\right)$ we obtain

$$
A_{k-1}\left(v_{k}^{k}\left(\omega^{k}\right) \wedge A_{-1}^{-1}\left(\omega^{-1}\right)\right) \equiv T\left(\omega^{-1} \wedge \omega^{k}\right) \quad\left(\bmod . \omega^{-1} \wedge \omega^{-1}, \omega^{0}, \ldots, \omega^{k-1}\right) .
$$

Substituting $\tau\left(X_{-1}\right) \wedge \tau\left(X_{k}\right), X_{-1} \in V_{-1}, X_{k} \in V_{k}^{0}$ to this equation, we have

$$
A_{k-1}\left(v_{k}^{k}\left(X_{k}\right) \wedge A_{-1}^{-1}\left(X_{-1}\right)\right)=T\left(X_{-1}, X_{k}\right) .
$$

If $v_{k}^{k}\left(X_{k}\right)=0$, then $T\left(X_{-1}, X_{k}\right)=0$ for any $X_{-1} \in V_{-1}$. From condition $\left(\mathrm{C}_{1}\right)$ we obtain $X_{k}=0$. Hence $v_{k}^{k}(x) \in \operatorname{Hom}\left(V_{k}^{0}, V_{k}\right)$ is injective for any $x \in R_{k}$. Set $\widetilde{\rho}=\rho^{k} \circ F$. By the definition of $F$ we have

$$
u^{p} \circ \widetilde{\rho}=u^{p} \circ \rho^{k} \circ F=u^{p} \circ F=v^{p} \quad(-1 \leq p \leq k-1) .
$$

This relation and (1) of the proposition $\left(\mathrm{P}_{k}\right)$ imply that $\widetilde{\rho}$ is a submersion. Therefore $F$ determines locally a local differential equation of order $k$.

Remark 4.1 By virtue of this theorem, a system $\left(R_{k}, W_{k}^{0}, T, \omega\right)$ satisfying the conditions stated in the theorem may be also called a local differential equation of order $k$.

\section{Equivalence}

Let $V_{k}^{0}$ be a subspace of $V_{k}(k \geq 1)$. We set $W_{k}^{0}=V_{-1} \oplus V_{0} \oplus \cdots \oplus V_{k}^{0}$ and $D^{p}=$ $V_{p} \oplus V_{p-1} \oplus \cdots \oplus V_{k}^{0}, p=0,1, \ldots, k$. We define the Lie subgroup $\mathrm{G}\left(W_{k}^{0}\right)$ of $\mathrm{GL}\left(W_{k}\left(V_{-1}, V_{0}\right)\right)$ as follows:

$$
\mathrm{G}\left(W_{k}^{0}\right)=\left\{g \in \mathrm{GL}\left(W_{k}\left(V_{-1}, V_{0}\right)\right) \mid g\left(V_{-1} \oplus V_{k}^{0}\right)=V_{-1} \oplus V_{k}^{0}, g\left(D^{p}\right)=D^{p}(0 \leq p \leq k)\right\} .
$$


Definition 5.1 We shall say that two local differential equations $\left(R_{k}, W_{k}^{0}, T, \omega\right)$ and $\left(R_{k}, W_{k}^{0}, T^{\prime}, \omega^{\prime}\right)$ are structurally equivalent if there exists a differentiable mapping $A: R_{k} \rightarrow \mathrm{G}\left(W_{k}^{0}\right)$ such that $\omega^{\prime}=A(\omega)$.

Definition 5.2 A local differential equations $\left(R_{k}, W_{k}^{0}, T, \omega\right)$ will be said of type $W_{k}^{0}$ if $T_{x}\left(X_{-1}, X_{k}\right)=\left[X_{-1}, X_{k}\right]_{0}$ for all $x \in R_{k}, X_{-1} \in V_{-1}, X_{k} \in V_{k}^{0}$ and if $\omega$ satisfies the condition:

$\left(C_{2}^{\prime}\right) \quad$ (i) $d \omega^{-1} \equiv 0\left(\bmod . \omega^{-1}\right)$;

(ii) For $p=0,1, \ldots, k-1$,

$$
d \omega^{p}+\left[\omega^{-1} \wedge \omega^{p-1}\right] \equiv 0 \quad\left(\bmod . \omega^{0}, \ldots, \omega^{p}\right),
$$

where $[\cdot, \cdot]_{0}$ denotes the natural bracket operation $W_{k}^{0}$ (cf. Proposition 4.1$)$.

Remark 5.1 Almost all local differential equations which admit a lot of solutions turn out to be structurally equivalent to a local differential equation of type $W_{k}^{0}$ for some $V_{k}^{0}$.

Example 5.1 Take the system of second order differential equations

$$
\frac{\partial^{2} z}{\partial x^{2}}=0 \quad, \quad \frac{\partial^{2} z}{\partial x \partial y}=z .
$$

Putting on $\mathbb{R}^{6}=\{(x, y, z, p, q, t)\} \omega^{1}=d x, \omega^{2}=d y, \omega^{3}=d z-p d x-q d y, \omega^{4}=d p-z d y$, $\omega^{5}=d q-z d x-t d y$ and $\omega^{6}=d t$, we have

$$
\left\{\begin{array}{l}
d \omega^{1}=0, d \omega^{2} \equiv 0 \\
d \omega^{3}=\omega^{1} \wedge \omega^{4}+\omega^{2} \wedge \omega^{5} \\
d \omega^{4}=\omega^{2} \wedge \omega^{3}+p \omega^{1} \wedge \omega^{2} \\
d \omega^{5}=\omega^{2} \wedge \omega^{6}-\omega^{1} \wedge \omega^{3}-q \omega^{1} \wedge \omega^{2} \\
d \omega^{6}=0 .
\end{array}\right.
$$

Since one can not remove the terms $p \omega^{1} \wedge \omega^{2}$ and $q \omega^{1} \wedge \omega^{2}, \omega$ is not of type $W_{2}^{0}$ for any $V_{2}^{0} \subset V_{2}$. On the other hand, the given system (5.1) has no solutions except $z=0$.

Proposition 5.1 Let $P$ be a differentiable manifold with $\operatorname{dim} P \geq \operatorname{dim} W_{k}^{0}$. Suppose there exists a $W_{k}^{0}$-valued 1-form $\omega=\omega^{-1}+\omega^{0}+\cdots+\omega^{k}$ such that $\omega_{p}: T_{p}(P) \rightarrow W_{k}^{0}$ is surjective for any $p \in P$ and $d \omega \equiv 0$ (mod. $\omega$ ). If $\omega$ satisfies the condition $\left(C_{2}^{\prime}\right)$ in Definition 5.2. then $\left(P, W_{k}^{0}, \omega\right)$ determines locally a local differential equation of type $W_{k}^{0}$.

Proof. Since $\omega=0$ is completely integrable, there exists, for each $p \in P$, an open neighborhood $U$ of $p$, a differentiable manifold $R_{k}$ with $\operatorname{dim} R_{k}=\operatorname{dim} W_{k}^{0}$ and a fibering $\pi: U \rightarrow R_{k}$ such that each fiber is a maximal integral manifold of $\left.\omega\right|_{U}=0$. Let $\sigma: R_{k} \rightarrow U$ be a differentiable cross section of $\pi$ and put $\bar{\omega}=\sigma^{*} \omega$. Then it is clear that $\left(R_{k}, W_{k}^{0}, \bar{\omega}\right)$ is a local differential equation of type $W_{k}^{0}$. In general, the obtained system depends on the choice of cross sections. 
For a subspace $V_{k}^{0}$ of $V_{k}$ we set

$$
\left(V_{k}^{0}\right)^{(1)}=\left\{X \in \operatorname{Hom}\left(V_{-1}, V_{k}^{0}\right) \mid X(u)(v)=X(v)(u), u, v \in V_{-1}\right\} .
$$

For a subspace $U$ of $V_{-1}$, we set

$$
V_{k}^{0}(U)=\left\{X_{k} \in V_{k}^{0} \mid X_{k}(u)=0, u \in U\right\} .
$$

Definition 5.3 A subspace $V_{k}^{0}$ of $V_{k}$ is said involutive if there exists a series of subspaces (0) $\subset U_{0} \subset U_{1} \subset \cdots \subset U_{n-1} \subset U_{n}=V_{-1}$ with $\operatorname{dim} U_{i}=i$ such that

$$
\operatorname{dim}\left(V_{k}^{0}\right)^{(1)}=\sum_{i=0}^{n} \operatorname{dim} V_{k}^{0}\left(U_{i}\right) .
$$

A local differential equation $\left(R_{k}, W_{k}^{0}, \omega\right)$ of type $W_{k}^{0}$ is said involutive if $V_{k}^{0}$ is involutive.

Example 5.2 We use the notation of Example 4.1. By the symbol $U=\left(X_{1}, X_{2}, \ldots, X_{n}\right)$ we mean that the vector space $U$ is spanned by the basis $X_{1}, X_{2}, \ldots, X_{n}$.

(1) $\operatorname{dim} V_{-1}=1, \operatorname{dim} V_{0}=1$.

(i) $k=1$. Then $W_{1}^{0}=V_{-1} \oplus V_{0}=\left(X_{1}, X_{2}\right)$ with $\left[X_{1}, X_{2}\right]=0$. A $W_{1}^{0}$-valued 1 -form $\omega=\omega^{1} X_{1}+\omega^{2} X_{2}$ is of type $W_{1}^{0}$ if it satisfies

$$
\begin{cases}d \omega^{1} \equiv 0 & \left(\bmod \cdot \omega^{1}\right) \\ d \omega^{2} \equiv 0 & \left(\bmod \cdot \omega^{2}\right)\end{cases}
$$

(ii) $k=2$. Then $W_{2}^{0}=V_{-1} \oplus V_{0} \oplus V_{1}=\left(X_{1}, X_{2}, X_{3}\right)$ with $\left[X_{1}, X_{3}\right]=-X_{2}$ and otherwise $\left[X_{i}, X_{j}\right]=0 . \omega=\omega^{1} X_{1}+\omega^{2} X_{2}+\omega^{3} X_{3}$ is of type $W_{2}^{0}$ if it satisfies

$$
\left\{\begin{array}{l}
d \omega^{1} \equiv 0 \quad\left(\bmod . \omega^{1}\right), \\
d \omega^{2}=\omega^{1} \wedge \omega^{3} \quad\left(\bmod \omega^{2}\right) \\
d \omega^{3}=0 \quad\left(\bmod . \omega^{2}, \omega^{3}\right) \\
\cdot
\end{array}\right.
$$

The Pfaffian system $\Sigma=\left\langle\omega^{2}, \omega^{3}\right\rangle$ determines a family of second-ordeer ordinary differential equations.

(2) $\operatorname{dim} V_{-1}=2, \operatorname{dim} V_{0}=1$.

(i) $k=1$. Let $V_{1}^{0}$ be a 1-dimensional subspace of $V_{1}$.

The we can choose a basis $X_{1}, X_{2}, X_{3}, X_{4}$ of $W_{1}^{0}$ such that $X_{1}, X_{2} \in V_{-1}$ and $\left[X_{2}, X_{4}\right]=-X_{3}$ and otherwise $\left[X_{i}, X_{j}\right]=0 . \omega=\sum_{i=1}^{4} \omega^{i} X_{i}$ is of type $W_{1}^{0}$ if it satisfies

$$
\left\{\begin{array}{l}
d \omega^{1} \equiv 0, d \omega^{2} \equiv 0 \quad\left(\bmod . \omega^{1}, \omega^{2}\right) \\
d \omega^{3} \equiv \omega^{2} \wedge \omega^{4} \quad\left(\bmod . \omega^{3}\right) .
\end{array}\right.
$$

We have already seen this structure equation in Section 3 , 
(ii) $k=2$. Let $V_{2}^{0}$ be a 2-dimensional subspace of $V_{2}$. Then the Lie algebra $W_{2}^{0}$ is isomorphic to the following three Lie algebras.

(a) $W_{2}^{0}=\left(X_{1}, X_{2}, X_{3}, X_{4}, X_{5}, X_{6}, X_{7}\right)$ with

$$
\begin{array}{lllll}
{\left[X_{1}, X_{4}\right]=-X_{3}} & , & {\left[X_{2}, X_{5}\right]=-X_{3}} & , & {\left[X_{1}, X_{6}\right]=-X_{4}} \\
{\left[X_{1}, X_{7}\right]=-X_{5}} & , & {\left[X_{2}, X_{7}\right]=-X_{4}} & , & {\left[X_{2}, X_{6}\right]=X_{5}}
\end{array}
$$

and otherwise $\left[X_{i}, X_{j}\right]=0 ; \omega=\sum_{i=1}^{7} \omega^{i} X_{i}$ is of type $W_{2}^{0}$ if it satisfies

$$
\begin{cases}d \omega^{1} \equiv 0, d \omega^{2} \equiv 0 \quad\left(\bmod . \omega^{1}, \omega^{2}\right) \\ d \omega^{3} \equiv \omega^{1} \wedge \omega^{4}+\omega^{2} \wedge \omega^{5} & \left(\bmod . \omega^{3}\right) \\ d \omega^{4} \equiv \omega^{1} \wedge \omega^{6}+\omega^{2} \wedge \omega^{7} & \left(\bmod . \omega^{3}, \omega^{4}, \omega^{5}\right) \\ d \omega^{5} \equiv \omega^{1} \wedge \omega^{7}-\omega^{2} \wedge \omega^{6} & \left(\bmod . \omega^{3}, \omega^{4}, \omega^{5}\right) .\end{cases}
$$

(b) $W_{2}^{0}=\left(X_{1}, X_{2}, X_{3}, X_{4}, X_{5}, X_{6}, X_{7}\right)$ with

$$
\begin{array}{lll}
{\left[X_{1}, X_{4}\right]=-X_{3}} & , & {\left[X_{2}, X_{5}\right]=-X_{3}} \\
{\left[X_{1}, X_{6}\right]=-X_{4}} & , & {\left[X_{2}, X_{7}\right]=-X_{5}}
\end{array}
$$

and otherwise $\left[X_{i}, X_{j}\right]=0 ; \omega=\sum_{i=1}^{7} \omega^{i} X_{i}$ is of type $W_{2}^{0}$ if it satisfies

$$
\left\{\begin{array}{l}
d \omega^{1} \equiv 0, d \omega^{2} \equiv 0 \quad\left(\bmod . \omega^{1}, \omega^{2}\right) \\
d \omega^{3} \equiv \omega^{1} \wedge \omega^{4}+\omega^{2} \wedge \omega^{5} \quad\left(\bmod . \omega^{3}\right) \\
d \omega^{4} \equiv \omega^{1} \wedge \omega^{6} \quad\left(\bmod \cdot \omega^{3}, \omega^{4}, \omega^{5}\right) \\
d \omega^{5} \equiv \omega^{2} \wedge \omega^{7} \quad\left(\bmod \cdot \omega^{3}, \omega^{4}, \omega^{5}\right)
\end{array}\right.
$$

Let $V_{2}^{0}$ be a 1-dimensional involutive subspace of $V_{2}$. Then there is only one case up to isomorphic algebra.

(c) $W_{2}^{0}=\left(X_{1}, X_{2}, X_{3}, X_{4}, X_{5}, X_{6}\right)$ with

$$
\left[X_{1}, X_{4}\right]=-X_{3} \quad, \quad\left[X_{2}, X_{5}\right]=-X_{3} \quad, \quad\left[X_{2}, X_{6}\right]=-X_{5}
$$

and otherwise $\left[X_{i}, X_{j}\right]=0 ; \omega=\sum_{i=1}^{6} \omega^{i} X_{i}$ is of type $W_{2}^{0}$ if it satisfies

$$
\left\{\begin{array}{l}
d \omega^{1} \equiv 0, d \omega^{2} \equiv 0 \quad\left(\bmod . \omega^{1}, \omega^{2}\right) \\
d \omega^{3} \equiv \omega^{1} \wedge \omega^{4}+\omega^{2} \wedge \omega^{5} \quad\left(\bmod . \omega^{3}\right) \\
d \omega^{4} \equiv 0 \quad\left(\bmod . \omega^{3}, \omega^{4}, \omega^{5}\right) \\
d \omega^{5} \equiv \omega^{2} \wedge \omega^{6} \quad\left(\bmod . \omega^{3}, \omega^{4}, \omega^{5}\right)
\end{array}\right.
$$

We have already discussed this case in Section 3.

\section{Lie algebraic aspects of differential equations}

In this section we shall consider the Lie-algebraic aspect of local differential equations. Let $V_{k}^{0}$ be a subspace of $V_{k}$. We set

$$
W_{k}^{0}\left(V_{k}^{0}\right)=V_{-1} \oplus V_{0} \oplus \cdots \oplus V_{k}^{0} \quad, \quad D^{p}=V_{p} \oplus V_{p-1} \oplus \cdots \oplus V_{k}^{0} \quad(0 \leq p \leq k) \text {. }
$$


We define the Lie algebra $\mathcal{J}\left(W_{k}^{0}\left(V_{k}^{0}\right)\right)$ as follows:

$\mathcal{J}_{k}\left(W_{k}^{0}\left(V_{k}^{0}\right)\right)=\left\{X \in \operatorname{gl}\left(W_{k}^{0}\left(V_{k}^{0}\right)\right) \mid X\left(V_{-1} \oplus V_{k}^{0}\right) \subset V_{-1} \oplus V_{k}^{0}, X\left(D^{p}\right) \subset D^{p}(0 \leq p \leq k)\right\}$.

Every element of $\mathcal{J}_{k}\left(W_{k}^{0}\left(V_{k}^{0}\right)\right)$ is of the form:

$$
\begin{gathered}
V_{-1} V_{0} V_{1} \cdots V_{k-1} V_{k}^{0} \\
\left(\begin{array}{cccccc}
* & 0 & 0 & \cdots & 0 & 0 \\
0 & * & 0 & \cdots & 0 & 0 \\
0 & * & * & \cdots & 0 & 0 \\
\vdots & \vdots & \vdots & \ddots & & \vdots \\
0 & * & * & \cdots & * & 0 \\
& * & * & \cdots & * & *
\end{array}\right) \\
V_{-1} \\
V_{1} \\
V_{k-1} \\
V_{k}^{0}
\end{gathered}
$$

where $*$ denotes a certain matrix. We define a mapping $\partial: \operatorname{Hom}\left(W_{k}^{0}, \mathcal{J}_{k}\left(W_{k}^{0}\right)\right) \rightarrow$ $\operatorname{Hom}\left(W_{k}^{0}, \wedge W_{k}^{0}: W_{k}^{0}\right)$ by the formula

$$
(\partial S)(X \wedge Y)=S(X)(Y)-S(Y)(X)
$$

for all $S \in \operatorname{Hom}\left(W_{k}^{0}, \mathcal{J}_{k}\left(W_{k}^{0}\right)\right)$ and all $X, Y \in W_{k}^{0}$, where we put $W_{k}^{0}=W_{k}^{0}\left(V_{k}^{0}\right)$.

Definition 6.1 Let $\mathcal{J}$ be a Lie algebra. A system $\left(\mathcal{J}, \mathcal{M}, \mathcal{J}_{0}\right)$ will be called a differential Lie algebra if it satisfies the following conditions:

(1) $\mathcal{J}_{0}$ is a Lie subalgebra of $\mathcal{J}$ and $\mathcal{M}$ is a vector subspace of $\mathcal{J}$ such that $\mathcal{J}=\mathcal{M} \oplus \mathcal{J}_{0}$ (direct sum);

(2) For some subspaces $V_{-1}, V_{0}$ and $V_{k}^{0}$ of $\mathcal{M}$, we have $\mathcal{M}=W_{k}^{0}\left(V_{k}^{0}\right)$;

(3) The linear isotropy representation $\rho: \mathcal{J}_{0} \rightarrow \operatorname{gl}(\mathcal{M})\left(\rho\left(X_{0}\right)(X)=\right.$ the $m$-component of $\left.\left[X_{0}, X\right], X_{0} \in \mathcal{J}_{0}, X \in \mathcal{M}\right)$ has its values in $\mathcal{J}_{k}\left(W_{k}^{0}\left(V_{k}^{0}\right)\right)$;

(4) Let $\alpha: \mathcal{M} \wedge \mathcal{M} \rightarrow \mathcal{M}$ be the linear mapping defined by the formula

$$
\alpha(X \wedge Y)=\text { the m-component of }[X, Y] \quad, \quad X, Y \in \mathcal{M} .
$$

There exists an element $S \in \operatorname{Hom}\left(\mathcal{M}, \mathcal{J}_{k}\left(W_{k}^{0}\right)\right)$ such that

$$
\alpha(X \wedge Y)=[X, Y]_{0}+(\partial S)(X \wedge Y)
$$

where $[,]_{0}$ denotes the natural bracket operation on $W_{k}^{0}\left(V_{k}^{0}\right)$. If $J_{0}=(0), \mathcal{J}(=\mathcal{M})$ will be called fundamental.

Theorem 6.1 Let $\left(\mathcal{J}, \mathcal{M}, \mathcal{J}_{0}\right)$ be a differential Lie algebra. Let $G$ be a Lie group corresponding to the Lie algebra $\mathcal{J}$ and let $\theta$ be the Maurer-Cartan form of $G$. Then the $\mathcal{M}$ component $\omega$ of $\theta$ with respect to the decomposition $\mathcal{J}=\mathcal{M} \oplus \mathcal{J}_{0}$ determines locally a local differential equation. 
Proof. Let $\mathcal{M}=W_{k}^{0}\left(V_{k}^{0}\right)$ for the subspaces $V_{-1}, V_{0}$ and $V_{k}^{0}$ of $\mathcal{M}$. We set $\theta=\omega+\omega_{0}$, $\omega_{0}$ being the $\mathcal{J}_{0}$-component of $\theta$. Then from the structure equation of Maurer-Cartan we obtain

$$
d \omega+\frac{1}{2} \alpha(\omega \wedge \omega)-\rho\left(\omega_{0}\right) \wedge \omega=0
$$

and hence

$$
d \omega+\frac{1}{2}[\omega \wedge \omega]_{0}+\frac{1}{2}(\partial S)(\omega \wedge \omega)-\rho\left(\omega_{0}\right) \wedge \omega=0 .
$$

Since $S(X)$ and $\rho\left(X_{0}\right)\left(X \in \mathcal{M}, X_{0} \in \mathcal{J}_{0}\right)$ lie in $\mathcal{J}_{k}\left(W_{k}^{0}\right)$, we have

$$
d \omega^{-1} \equiv 0 \quad\left(\bmod . \omega^{-1}\right.
$$

and for $p=0,1, \ldots, k-1$

$$
d \omega^{p}+\left[\omega^{-1} \wedge \omega^{p-1}\right]_{0} \equiv 0 \quad\left(\bmod \cdot \omega^{0}, \omega^{1}, \ldots, \omega^{p}\right)
$$

Therefore the theorem follows from Proposition 5.1.

Example 6.1 We define the 6-dimensional Lie algebra $\mathcal{J}=\left(X_{1}, X_{2}, X_{3}, X_{4}, X_{5}, X_{6}\right)$ by the following bracket operations:

$$
\begin{aligned}
& {\left[X_{1}, X_{4}\right]=-X_{2},\left[X_{1}, X_{5}\right]=-X_{1},\left[X_{2}, X_{4}\right]=-X_{3},\left[X_{2}, X_{5}\right]=-X_{2}} \\
& {\left[X_{2}, X_{6}\right]=-X_{2},\left[X_{3}, X_{5}\right]=-X_{3},\left[X_{3}, X_{6}\right]=-2 X_{3},\left[X_{4}, X_{6}\right]=-X_{4}}
\end{aligned}
$$

and otherwise $\left[X_{i}, X_{j}\right]=0$. Set $\mathcal{M}=\left(X_{1}, X_{2}, X_{3}, X_{4}\right)$ and $\mathcal{J}_{0}=\left(X_{5}, X_{6}\right)$. Then $\left(\mathcal{J}, \mathcal{M}, \mathcal{J}_{0}\right)$ is a differential Lie algebra of order 1 . Let $G$ be a connected Lie group corresponding to the Lie algebra $\mathcal{J}$ and let $\omega$ be the Maurer-Cartan form of $G$. Setting $\omega=\sum_{i=1}^{6} \omega^{i} X_{i}$ we have

$$
\left\{\begin{array}{l}
d \omega^{1}=\omega^{1} \wedge \omega^{5} \\
d \omega^{2}=\omega^{1} \wedge \omega^{4}+\omega^{2} \wedge\left(\omega^{5}+\omega^{6}\right) \\
d \omega^{3}=\omega^{2} \wedge \omega^{4}+\omega^{3} \wedge\left(\omega^{5}+2 \omega^{6}\right) \\
d \omega^{4}=\omega^{4} \wedge \omega^{6} \\
d \omega^{5}=0 \\
d \omega^{6}=0
\end{array}\right.
$$

The differential equation (3.1) considered in Section 3 belongs to this homogeneous case. The differential equation is left invariant by the Lie group, which can be considered as a subgroup of the contact transformation group.

Theorem 6.2 If $\mathcal{J}=\mathcal{J}_{-1}+\mathcal{J}_{0}+\mathcal{J}_{1}$ (direct sum) is a semi-simple graded Lie algebra, i.e. $\left[\mathcal{J}_{i}, \mathcal{J}_{j}\right] \subset \mathcal{J}_{i+j}(i, j=0, \pm 1, \pm 2, \ldots)$, where we put $\mathcal{J}_{p}=(0)$ for $p \leq-2$ and $p \geq 2$, then $\mathcal{J}$ has a structure of fundamental differential Lie algebra. 
Proof. Let $B$ be the Killinng-Cartan form of $\mathcal{J}$. The linear endomorphism $s$ of $\mathcal{J}$ defined by

$$
s\left(X_{-1}+X_{0}+X_{1}\right)=-X_{-1}+X_{0}-X_{1} \quad\left(X_{-1} \in \mathcal{J}_{-1}, X_{0} \in \mathcal{J}_{0}, X_{1} \in \mathcal{J}_{1}\right)
$$

is an involutive automorphism of $\mathcal{J}$. Hence

$B\left(X_{1}, X_{0}\right)=B\left(s\left(X_{1}\right), s\left(X_{0}\right)\right)=B\left(-X_{1}, X_{0}\right)=-B\left(X_{1}, X_{0}\right) \quad\left(X_{0} \in \mathcal{J}_{0}, X_{1} \in \mathcal{J}_{1}\right)$

Therefore we have

$$
B\left(\mathcal{J}_{1}, \mathcal{J}_{0}\right)=0
$$

Let $X_{1} \in \mathcal{J}_{1}$ be an element satisfying $\left[X_{1}, \mathcal{J}_{-1}\right]=(0)$. For $Y_{-1} \in \mathcal{J}_{-1}$ and $Z_{i} \in \mathcal{J}_{i}$ $(i=-1,0,1)$, we have $\operatorname{ad}\left(X_{1}\right) \circ \operatorname{ad}\left(Y_{-1}\right)\left(Z_{-1}\right)=0$,

$$
\operatorname{ad}\left(X_{1}\right) \circ \operatorname{ad}\left(Y_{-1}\right)\left(Z_{0}\right)=\left[X_{1},\left[Y_{-1}, Z_{0}\right]\right]=0
$$

and

$$
\operatorname{ad}\left(X_{1}\right) \circ \operatorname{ad}\left(Y_{-1}\right)\left(Z_{1}\right)=-\left[Y_{1},\left[Z_{1}, X_{1}\right]\right]-\left[Z_{1},\left[X_{1}, Y_{-1}\right]\right]=0
$$

Hence

$$
B\left(X_{1}, \mathcal{J}_{-1}\right)=0
$$

For $Y_{1} \in \mathcal{J}_{1}$ and $Z_{i} \in \mathcal{J}_{i}(i=-1,0,1)$, we have

$$
\operatorname{ad}\left(X_{1}\right) \circ \operatorname{ad}\left(Y_{1}\right)\left(Z_{-1}\right)=-\left[Y_{1},\left[Z_{-1}, X_{1}\right]\right]-\left[Z_{-1},\left[X_{1}, Y_{1}\right]\right]=0
$$

$\operatorname{ad}\left(X_{1}\right) \circ \operatorname{ad}\left(Y_{1}\right)\left(Z_{0}\right)=0$ and $\operatorname{ad}\left(X_{1}\right) \circ \operatorname{ad}\left(Y_{1}\right)\left(Z_{1}\right)=0$. Hence

$$
B\left(X_{1}, \mathcal{J}_{0}\right)=0
$$

These three relations (6.1), (6.2) and (6.3) yield $B\left(X_{1}, \mathcal{J}\right)=0$. Since $\mathcal{J}$ is semi-simple, this implies $X_{1}=0$. Therefore $\mathcal{J}_{1}$ can be considered a subspace of $\operatorname{Hom}\left(\mathcal{J}_{-1}, \mathcal{J}_{0}\right)$ by the mapping $h: \mathcal{J}_{1} \rightarrow \operatorname{Hom}\left(\mathcal{J}_{-1}, \mathcal{J}_{0}\right)$ defined by $h\left(X_{1}\right)\left(X_{-1}\right)=\left[X_{1}, X_{-1}\right], X_{1} \in \mathcal{J}_{1}$, $X_{-1} \in \mathcal{J}_{-1}$. Next, we define the element $s \in \operatorname{Hom}\left(\mathcal{J}, \mathcal{J}_{1}\left(W_{1}^{0}\left(\mathcal{J}_{1}\right)\right)\right)$ by the formulas:

$$
\begin{aligned}
& S\left(X_{-1}\right)=0 \quad, \quad S\left(X_{1}\right)=0 \quad, \quad S\left(X_{0}\right)\left(Y_{-1}\right)=\left[X_{0}, Y_{-1}\right] \\
& S\left(X_{0}\right)\left(Y_{0}\right)=\frac{1}{2}\left[X_{0}, Y_{0}\right] \quad, \quad S\left(X_{0}\right)\left(Y_{1}\right)=\left[X_{0}, Y_{1}\right]
\end{aligned}
$$

$X_{-1}, Y_{-1} \in \mathcal{J}_{-1}, X_{0}, Y_{0} \in \mathcal{J}_{0}, X_{1}, Y_{1} \in \mathcal{J}_{1}$. Then we have

$$
[X, Y]=[X, Y]_{0}+(\partial S)(X \wedge Y)
$$

for all $X, Y \in \mathcal{J}$.

The simple real Lie algebras having the structure stated in Theorem 6.2 are classified in S. Kobayashi and T. Nagano [1]. Among these simple Lie algebras $\operatorname{sl}(2: \mathbb{R})$ is the 
simplest example. Moreover $\operatorname{sl}(2: \mathbb{R})$ has the structure of a differential Lie algebra which is not fundamental. Set

$$
\mathcal{M}=\left\{\left(\begin{array}{ll}
0 & a \\
b & 0
\end{array}\right) \mid a, b \in \mathbb{R}\right\} \quad, \quad \mathcal{J}_{o}=\left\{\left(\begin{array}{cc}
u & 0 \\
0 & -u
\end{array}\right) \mid u \in \mathbb{R}\right\} .
$$

$\left(\operatorname{sl}(2: \mathbb{R}), \mathcal{M}, \mathcal{J}_{0}\right)$ is a differential Lie algebra of order 1 . Let $\omega$ be the Maurer-Cartan form of $\mathrm{SL}(2: \mathbb{R})$ and set

$$
\omega=\omega^{1}\left(\begin{array}{ll}
0 & 1 \\
0 & 0
\end{array}\right)+\omega^{2}\left(\begin{array}{ll}
0 & 0 \\
1 & 0
\end{array}\right)-\omega^{3}\left(\begin{array}{cc}
0 & 1 \\
0 & -1
\end{array}\right) .
$$

We have the structure equation

$$
\left\{\begin{aligned}
d \omega^{1} & =-2 \omega^{1} \wedge \omega^{3} \\
d \omega^{2} & =2 \omega^{2} \wedge \omega^{3} \\
d \omega^{3} & =\omega^{1} \wedge \omega^{2}
\end{aligned}\right.
$$

For example, we can choose $\omega^{1}, \omega^{2}, \omega^{3}$ as follows:

$$
\left\{\begin{array}{l}
\omega^{1}=\mathrm{e}^{2 z} d x \\
\omega^{2}=\mathrm{e}^{-2 z}\left(d y-\left(y^{2}+a(x) y+b(x)\right) d x\right) \\
\omega^{3}=d z-\left(y+\frac{1}{2} a(x)\right) d x
\end{array}\right.
$$

where $a(x)$ and $b(x)$ denote two arbitrary differentiable functions of the variable $x$. Therefore we can see that $\mathrm{SL}(2: \mathbb{R})$ corresponds to the family of ordinary differential equations of Riccati type.

\section{Cartan example}

Now we can consider the involutive system of second-order differential equations which is one of the typical examples in Cartn's paper [6]:

$$
\frac{\partial^{2} z}{\partial x^{2}}=\frac{1}{3}\left(\frac{\partial^{2} z}{\partial y^{2}}\right)^{3} \quad, \quad \frac{\partial^{2} z}{\partial x \partial y}=\frac{1}{2}\left(\frac{\partial^{2} z}{\partial y^{2}}\right)^{2} .
$$

Setting on $\mathbb{R}^{6}=\{(x, y, z, p, q, t)\} \omega^{1}=d x, \omega^{2}=d y+t d x, \omega^{3}=d z-p d x-q d y$, $\omega^{4}=d p-t d q+\frac{1}{6} t^{3} d x+\frac{1}{2} t^{2} d y, \omega^{5}=d q-\frac{1}{2} t^{2} d x-t d y$ and $\omega^{6}=d t$, we have the structure equations of the system:

$$
\left\{\begin{array}{l}
d \omega^{1}=0 \\
d \omega^{2}=-\omega^{1} \wedge \omega^{6} \\
d \omega^{3}=\omega^{1} \wedge \omega^{4}+\omega^{2} \wedge \omega^{5} \\
d \omega^{4}=\omega^{5} \wedge \omega^{6} \\
d \omega^{5}=\omega^{2} \wedge \omega^{6} \\
d \omega^{6}=0
\end{array}\right.
$$

which is of type $W_{2}^{0}$ inn Example $5.2(2)(\mathrm{ii})(\mathrm{c})$. Th integration of the system is deeply related to the structure equation (17.2). It is easy to see that $\left(\omega^{6}, \omega^{2}, \omega^{5}, \omega^{4}, \omega^{3}\right)$ forms 
a solvable system of the Pfaffian system $\Sigma=\left\langle\omega^{3}, \omega^{4}, \omega^{5}\right\rangle$. Therefore we can obtain by quadrature five independent first integrals of the Cauchy characteristic system of $\Sigma$ :

$$
\begin{aligned}
& u_{1}=z-x p+x q t+\frac{1}{6} x^{2} t^{3} \quad, \quad u_{2}=p-q t+\frac{1}{6} x t^{3}+\frac{1}{2} y t^{2} \\
& u_{3}=q-\frac{1}{2} y t^{2} \quad, \quad u_{4}=y+x t \quad, \quad u_{5}=t
\end{aligned}
$$

so that the system $\Sigma$ is expressed as

$$
\left\{\begin{aligned}
\omega^{3}-x \omega^{4} & =d u_{1}-\left(u_{3}+u_{4} u_{5}\right) d u_{4} \\
\omega^{4} & =d u_{2}+u_{3} d u_{5} \\
\omega^{5} & =d u_{3}+u_{4} d u_{5}
\end{aligned}\right.
$$

By this expression, we can construct the general integral surfaces of the given system (see [6, §38]).

Let $G$ be the Lie subgroup of $\mathrm{GL}(6: \mathbb{R})$ consisting of matrices of the form

$$
\left(\begin{array}{llllll}
* & * & * & * & * & 0 \\
* & * & * & * & * & 0 \\
0 & 0 & * & 0 & 0 & 0 \\
0 & 0 & * & * & * & 0 \\
0 & 0 & * & * & * & 0 \\
* & * & * & * & * & *
\end{array}\right)
$$

where $*$ is an element of $\mathbb{R}$. Let $B_{G}$ be the $G$-structure defined by the dual frame of $\left(\omega^{1}, \omega^{2}, \ldots, \omega^{6}\right)$. Then a contact transformation leaving the equations (17.2) invariant induces an automorphism of this $G$-structure and vice versa. The structure group $G$ can be reduced to the Lie subgroup $G_{7}$ whose Lie algebra $\mathcal{J}_{7}$ is given as follows:

$\mathcal{J}_{7}=\left\{\left(\begin{array}{cccccc}a_{1}-a_{2} & -a_{3} & a_{4} & 0 & 0 & 0 \\ 0 & a_{1} & a_{5} & 0 & \frac{4}{3} a_{3} & 0 \\ 0 & 0 & 2 a_{1}+a_{2} & 0 & 0 & 0 \\ 0 & 0 & a_{6} & a_{1}+2 a_{2} & 0 & 0 \\ 0 & 0 & a_{7} & a_{3} & a_{1}+a_{2} & 0 \\ 0 & -a_{6} & 0 & -a_{5} & \frac{4}{3} a_{7} & a_{2}\end{array}\right), a_{i} \in \mathbb{R}, i=1,2, \ldots, 7\right\}$.

The usual prolongations of $\mathcal{J}_{7}$ satisfy $\operatorname{dim} \mathcal{J}_{7}^{(1)}=1$ and $\mathcal{J}_{7}^{(P)}=\{0\}(p \geq 2)$. Therefore the local automorphism group of the given system with respect to the group $G$ is of finite type.

The structure equation (17.2) determines the Lie algebra $\mathcal{M}=\left(X_{1}, X_{2}, X_{3}, X_{4}, X_{5}, X_{6}\right)$ with the bracket operation

$$
\begin{aligned}
& {\left[X_{1}, X_{4}\right]=-X_{3},\left[X_{1}, X_{6}\right]=-X_{2},\left[X_{2}, X_{5}\right]=-X_{3}} \\
& {\left[X_{2}, X_{6}\right]=-X_{5},\left[X_{5}, X_{6}\right]=-X_{4}}
\end{aligned}
$$

and otherwise $\left[X_{i}, X_{j}\right]=0$. This Lie algebra has a structure of fundamental graded Lie algebra in the sense of $\mathrm{N}$. Tanaka [16]. We set $\mathcal{J}_{-1}=\left(X_{1}, X_{6}\right), \mathcal{J}_{-2}=\left(X_{2}\right), \mathcal{J}_{-3}=\left(X_{5}\right)$, 
$\mathcal{J}_{-4}=\left(X_{4}\right), \mathcal{J}_{-5}=\left(X_{3}\right)$. Then $\mathcal{M}=\mathcal{J}_{-5}+\mathcal{J}_{-4}+\mathcal{J}_{-3}+\mathcal{J}_{-2}+\mathcal{J}_{-1}$ (direct sum) is a fundamental graded algebra of the 5th kind. The structure of the automorphism group with respect to the group $G$ is given by Tanaka's prolongation method [16]. We can verify that the graded Lie algebra $\mathcal{J}$ prolonged from $\mathcal{M}$ with respect to the Lie algebra of $G$ has the following structure:

(1) $\mathcal{J}=\sum_{p=-5}^{5} \mathcal{J}_{p}$ (direct sum);

(2) $\mathcal{J}_{0}$ is a Cartan subalgebra of $\mathcal{J}$;

(3) $\operatorname{dim} \mathcal{J}_{0}=2, \operatorname{dim} \mathcal{J}_{ \pm 1}=2$ and $\operatorname{dim} \mathcal{J}_{ \pm p}=p$ for $p=2,3,4,5$;

(4) $\mathcal{J}$ is isomorphic to the exceptional simple real Lie algebra of dimension 14.

In order to transform the involutive systems which admit this 14-dimensional simple Lie group of contact transformations to the standard form (7.1), we need the integration of a system of differential equations associated with the simple group.

\section{References}

[1] R. Bryant, S.S. Chern, R. Gardner, H. Goldshmidt, and P. Griffiths. Exterior differential systems. Springer-Verlag, N.Y., 1991.

[2] E. Cartan. Sur la réduction à sa forme canonique de la structure d'un groupe de transformations fini et continu. Amer. J. Math., 18:1-61, 1896.

[3] E. Cartan. Sur l'intégration des systèmes d'équations aux différentielles totales. Ann. Ec. Norm., 18:241-3111, 1901.

[4] E. Cartan. Sur l'intégration des systèmes différentiels complètement intégrables. C. R. Acad. Sc., 134:1415-1418, 1902.

[5] E. Cartan. Les sous-groupes des groupes continus de transformations. Ann. Ec. Norm., 25:57-194, 1908.

[6] E. Cartan. Les systèmes de Pfaff à cinq variables et les équations aux dérivées partielles du second ordre. Ann. Ec. Norm., 27:109-192, 1910.

[7] E. Cartan. Les systèmes différentiels extérieurs et leurs applications géométriques. Hermann, Paris, 1945.

[8] F. Estrabrook and Wahlquist H. Prolongation structures of nonlinear evolution equations. J. Math. Phys., 16:1-7, 1975.

[9] P. Griffiths. On Cartan method of Lie group and moving frame as applied to uniquenes and existence questions in differential geometry. Duke Math. J., 41:775$814,1974$. 
[10] Th. Ivey and J.M. Landesberg. Cartan for beginners: differential geometry via moving frames and exterior differential systems. A.M.S.,Graduate Studies,61, 2004.

[11] S. Kobayashi and S. Nagano. On filtred Lie algebras and geometric structures I. J. Math. Mech., 13:875-907, 1964.

[12] R. Montgomery. A tour of subriemannian geometries, their geodesics and applications., volume 91. A.M.S.,Math.Survey and Monographs, 2002.

[13] P. Olver. Symmetry, invariants and equivalence. Springer-Verlag, N.Y., 1995.

[14] S. Sternberg. Lectures on differential geometry. Prentice-Hall., 1964.

[15] O. Stomark. Lie's structural approach to PDE systems. Cambridge University Press., 2000.

[16] N. Tanaka. On differential systems, graded Lie algebra and pseudogroups. J. Math. Kyoto Univ., 10:1-82, 1970.

[17] I. Zelenko. Fundamental form and Cartan's tensor of (2,5)-distributions. Preprint, SISSA-Trieste., 2005. 